\title{
El estatuto de los bioartefactos. Intencionalismo, reproductivismo y naturaleza ${ }^{1}$
}

\author{
(The nature of bioartifacts. \\ Intentionalism, reproductivism, and nature)
}

\author{
Diego PARENTE
}

Recibido: 11 de enero de 2013

Aceptado: 8 de abril de 2013

\section{Resumen}

El presente trabajo aborda algunos aspectos destacados del problema ontológico de los bioartefactos con el objetivo de elaborar -en el interior del vocabulario de la filosofía de los artefactos técnicos- una noción deflacionada de bioartefacto que sea capaz de alumbrar una distinción valiosa entre aquellos procesos surgidos de una dinámica natural no intervenida intencionalmente y aquellos resultantes de una intervención intencional. Con este propósito se reconstruyen dos vías interpretativas sobre la naturaleza de dichos entes (intencionalismo y reproductivismo) para luego evaluar sus alcances y posibilidades. Finalmente este artículo recoge las críticas anteriores y procura cohesionarlas haciendo explícitos los niveles de intervención intencional y las condiciones de bioartefactualidad.

Palabras clave: Bioartefacto, artefacto técnico, intencionalismo, reproductivismo, naturaleza.

\begin{abstract}
This paper discusses some aspects of the ontological problem of bioartifacts in order to develop, within the vocabulary of philosophy of technical artifacts, a defla-

1 El presente trabajo fue realizado en el marco del proyecto Epistemología de los Artefactos. Affordances, conocimiento práctico y artefactos epistémicos, Ministerio de Ciencia e Innovación (España), número de referencia FF 12009-120054, proyecto dirigido por el Dr. Jesús Vega Encabo (Universidad Autónoma de Madrid, España).
\end{abstract}


tioned notion of bioartifact capable of revealing a meaningful distinction between those processes arisen from a natural dynamics not intentionally intervened, and those arisen from intentional intervention. With this purpose two ways of interpreting the nature of these entities (intentionalism and reproductivism) are reconstructed and evaluated. Finally this paper collects the previous arguments and tries to make explicit the levels of intentional intervention and the conditions to be a bioartifact.

Keywords: Bioartifact, technical artifact, intentionalism, reproductivism, nature.

¿Qué es aquello que otorga identidad a los bioartefactos? ¿Puede efectivamente hablarse de los bioartefactos como una clase bien definida sobre la cual sería legítimo trazar diferentes series de atribuciones funcionales y descripciones de propiedades? ¿O se trata, por el contrario, de una noción necesariamente volátil que debería evitarse desde el inicio en la medida en que bajo ella se alinean múltiples aporías ontológicas y epistemológicas?

Las respuestas a estos problemas en torno a la demarcación conceptual de los bioartefactos son heterogéneas. Como se verá, el puzzle teórico que implican es el de una tensión argumentativa entre intencionalismo y reproductivismo, dos enfoques habitualmente incompatibles en torno a la cuestión de la naturaleza de los artefactos y el despliegue de sus linajes. Esta tensión entre dos tipos de descripción es, en rigor, inherente a las condiciones del propio proceso de emergencia de los bioartefactos, esto es, un proceso caracterizado por estar sometido a agencia múltiple pero no traducible a los términos demiúrgicos del modelo hilemórfico; un proceso que responde a trayectorias biológicas autónomas de los organismos seleccionados, pero cuya direccionalidad está parcialmente apoyada en acciones intencionales que, a su vez, reflejan intereses humanos.

Con el objetivo de explicitar los motivos de esta tensión intentaré en lo que sigue reconstruir las líneas fundamentales de dos enfoques más o menos delimitados aclarando que esta distinción, sin embargo, no quita que algunas propuestas puedan efectivamente solaparse o entremezclarse. La tesis que se sugiere en este trabajo es que resulta valioso preservar una noción deflacionada de bioartefacto que sea capaz de dar cuenta de un conjunto cultural de intervenciones sobre aspectos inmanentes de lo viviente, esto es, un tipo de intervención que no deriva en la generación de artefactos prototípicos pero que, sin embargo, se encuentra atravesada por varios rasgos comunes a los de aquella.

La discusión del problema se halla estructurada de la siguiente manera. La sección [1] brinda algunas precisiones sobre el concepto de bioartefacto especialmente en lo concerniente a sus rasgos y al tipo de atribuciones funcionales que dichos 
entes soportan. La sección [2] intenta recomponer el puzzle que involucra el estatuto ontológico de dichas entidades. Con este propósito se reconstruyen dos posibles vías interpretativas al tiempo que se evalúan sus alcances y posibilidades. La sección [3] recoge las críticas anteriores y procura cohesionarlas haciendo explícitos los niveles de intervención intencional y las condiciones de bioartefactualidad. La sección [4], finalmente, realiza un racconto del recorrido teórico sugerido en el trabajo e indica sus conclusiones.

\section{Los bioartefactos: rasgos y condiciones}

Al menos desde el Neolítico, los humanos han desarrollado artefactos biológicos o "bioartefactos" a partir de la domesticación de una serie de plantas y animales. Dichos entes corporizan un complejo entramado en el cual se anudan funciones artefactuales y funciones biológicas, usos intencionales y procesos que transcurren independientemente de intervención humana. Pese a su relevancia en la historia de la cultura y la técnica, los bioartefactos no ocupan actualmente un lugar central en la discusión filosófica en torno a los artefactos. ${ }^{2}$

Los objetos naturales inanimados (una montaña, un río o una piedra) no involucran dificultades de atribución funcional en la medida en que sólo se les puede asignar funciones artefactuales en caso de que sean utilizados por agentes intencionales. Pero las aporías emergen una vez que introducimos en el análisis a los organismos, es decir, entes con autonomía constitutiva y con capacidad de auto-organización. Con ellos se abre una nueva dimensión de dificultades para establecer sus funciones dado que en el caso de algunos items biológicos uno estaría habilitado, al menos en principio, a predicar tanto funciones biológicas como funciones artefactuales.

Este repertorio de aporías se patentiza precisamente en los bioartefactos, es decir, en aquellas entidades apoyadas en soportes biológicos que han sido sometidas a selección artificial deliberada por parte de diseñadores humanos. Los procesos iniciales de domesticación que han dado lugar al surgimiento de bioartefactos han operado sobre ejemplares individuales seleccionando características observables tales como la presencia o ausencia de semillas o la altura de una planta, o el tamaño de los órganos o su masa muscular en el caso de los animales. ${ }^{3}$ Los bioartefactos realizan por sí mismos determinadas funciones biológicas que son cooptadas y adaptadas por agentes humanos para ciertos propósitos. Podría decirse

\footnotetext{
2 Los textos de Sperber (2007) y Longy (2009) son excepciones a esta regla.

3 Una interesante perspectiva sobre el despliegue histórico de los bioartefactos puede verse en Murphy (2007) y Newell-Mc Loughlin y Re (2006). En un artículo reciente, Cuevas (2008) presenta un enfoque filosófico sobre dichos procesos de domesticación.
} 
-siguiendo a Longy (2009)- que los humanos "sabotean" los mecanismos biológicos para sus propios fines.

A diferencia de otras especies biológicas, los bioartefactos poseen una "historia selectiva" en sentido propio, una historia intencional y no ciega. Son el producto de una serie de elecciones de animales o vegetales dotados de un conjunto de características relevantes para algún agente intencional. De tal modo, un bioartefacto (tal como la planta de cannabis o una especie determinada de perro) cumple su función artefactual realizando alguna de sus funciones biológicas. Si bien la biotecnología moderna agrega un mejor conocimiento de los procesos característicos de las acciones de domesticación, en todos estos casos los organismos tienen rasgos cuyas funciones resultan tanto de mecanismos naturales como de acciones intencionales humanas. ${ }^{4}$ Los bioartefactos poseen al menos una serie de rasgos que podrían ser denominados "artefactuales" dado que sin la intervención humana esos rasgos con dichos efectos intendidos no habrían aparecido (Longy 2009: p.56).

Es importante destacar que no todos los organismos biológicos insertados intencionalmente en planes de acción pueden ser legítimamente denominados "bioartefactos". Muchos entes vivientes son efectivamente usados como mediadores de una acción sin haber sido intencionalmente domesticados. Imaginemos -por ejemploque, en un día de camping, unos niños buscan un sapo cerca del arroyo para hacer un experimento un tanto cruel. El sapo finalmente elegido puede haber sido intencionalmente seleccionado, pero dicha selección no tiene permanencia en el tiempo, se trata de una conducta que no genera un linaje de prácticas de selección intencional de sapos con el objetivo $X$. Por el contrario, diríamos que es una práctica azarosamente guiada y sin posibilidades de estabilizarse socialmente. Sin embargo, sería contraintuitivo negar que el sapo haya sido utilizado: efectivamente fue usado aunque su uso no haya decantado en una práctica estable. En este sentido, el desafortunado sapo -al igual que un determinado pájaro elegido para probar un rifle- no pueden ser considerados bioartefactos.

Otros linajes de entidades naturales, tales como las sanguijuelas (Hirudo medicinalis), poseen una historia reproductiva más compleja y cambiante. Ciertamente su carácter de mediación técnica se revela en su misma denominación medicinalis, una clasificación obtenida a partir de su para-qué en el interior de una determinada práctica terapéutica. Este caso parece involucrar cierto uso estratégico de dichos organismos a través de la selección de ejemplares. De hecho, en cierto momento histórico de la cultura occidental se alcanzó una sofisticación importante en cuanto

\footnotetext{
4 Keekok Lee (2009) distingue tres grados de domesticación con complejidad creciente: (a) aquella producida por las técnicas artesanales de selección por ensayo y error; (b) aquellas técnicas de hibridización mendeliana que operan todavía a través del organismo completo de reproducción, pero no obstante se concentran en el cromosoma gen como unidad de transmisión genética; y (c) la genética de ADN cuya intervención opera en el nivel molecular.
} 
a su manipulación, el conocimiento de sus efectos y la cría controlada de dichos entes para usos terapéuticos relacionados con la extracción de sangre en pacientes. 5 Pero lo cierto es que aquello que se aprovecha de las sanguijuelas es una serie de capacidades -basadas, por supuesto, en al menos una función biológica- que se implantan convenientemente en un determinado plan de uso. A diferencia del ejemplo anterior (el sapo como mero intermediario ocasional), hallamos aquí un cierto grado de práctica y de criterio en la selección intencional de dichos organismos -selección que, no obstante, no llega a cristalizar en prácticas genuinas de domesticación-. En resumen, las sanguijuelas representan una suerte de categoría intermedia entre las simples mediaciones ocasionales y los bioartefactos auténticos.

Ahora bien, independientemente de casos fronterizos como el anterior, los argumentos desarrollados en esta sección tienden a mostrar que hay una esfera particular de organismos cuya peculiaridad reside en haber sido sometidos sistemáticamente a procesos de selección intencional que revelan intereses humanos singulares. En cuanto prácticas, hay algo que diferencia al uso ocasional de un pájaro -por parte de un tirador- como "objeto para probar la puntería" del hecho de sembrar un terreno después de haberlo preparado de manera apropiada, para luego cosechar y seleccionar los ejemplares más convenientes para el consumo. Se trata de una diferencia que, aunque no necesariamente genere estatutos ontológicos heterogéneos para el pájaro y el cereal, sí obliga al menos a reconocer que hay en juego dos prácticas distintas: una de ellas, amparada en una instrumentalidad básica; la otra, edificada sobre una racionalidad estratégica que organiza un proceso llevado adelante colectivamente, repetido y mantenido a través del tiempo.

La noción de bioartefacto defendida en este trabajo desafía un prejuicio esencial en la mayoría de los tratamientos filosóficos de la técnica y, especialmente, en las propuestas de raíz intencionalista: aquel que identifica lo artefactual con una unidad completa y terminada, inerte y heterónoma, es decir, un concepto modelado sobre la imagen de los útiles paleolíticos. ${ }^{6}$ La historia de la cultura muestra, sin embargo, que la interacción humana con bioartefactos es muy común desde el Neolítico. El proceso de domesticación que hizo emerger a los bioartefactos en dicho período puede ser leído, si se asume el punto de vista de dichos entes, como una coevolución que favoreció su propagación (o la de alguno de sus rasgos adaptativos) a nivel planetario. En este sentido, la cultura humana se ha adaptado a la biología del cereal tanto como los cereales se han adaptado a la cultura humana (Sperber 2007). Siguiendo esta línea de argumentación, si bien se suele enfatizar el papel activo y determinante de los agentes intencionales en el establecimiento de familias de bioartefactos, es preciso destacar que el mantenimiento de estas clases

\footnotetext{
5 Inclusive durante los siglos XVIII y XIX las sanguijuelas se vendían en las farmacias europeas, especialmente en Francia. Acerca de su uso histórico medicinal véase Vera, Blu y Torres (2005).

${ }^{6}$ Sobre esta crítica, véase Sperber (2007).
} 
en cuanto tales no puede ser explicado apelando exclusivamente a dichos agentes humanos. Como bien señala Longy (2009), en el caso de las especies domesticadas la estabilidad causal subyacente a las funciones bioartefactuales es el resultado de una compleja serie de fenómenos y mecanismos que actúan en distintos niveles: el modo en que las preferencias y el conocimiento humano transformaron la selección natural en artificial, el modo en el que los mecanismos culturales de varios tipos difundieron y aceleraron la selección artificial, el modo en que las plantas y animales explotaron las nuevas condiciones ambientales creadas por los humanos, etc (Longy 2009, p.59). ${ }^{7}$

\section{El puzzle planteado por los bioartefactos}

¿A qué región ontológica pertenecen los bioartefactos? ¿Son sencillamente seres naturales sobre los cuales equivocadamente se intenta proyectar cualidades artefactuales? ¿Se trata, por el contrario, de entes que caben bajo las condiciones que se aplican a cualquier artefacto tradicional? ¿O acaso constituyen una especie de tercer reino ontológico, recientemente inaugurado, el reino de lo híbrido? Inicialmente, situados en una indagación de orden ontológico, la noción de "bioartefacto" parece remitir a un referente compuesto por dos naturalezas distintas, una biológica o natural, y otra artificial, incorporada por alguna clase de acción técnica humana. Una vez admitido este rasgo, quizás podría pensarse que la clave de su interpretación radicaría en profundizar la idea de un "híbrido" (Fehér 1998) que integre naturaleza y artificio, componentes naturales y artificiales, una especie de cyborg en cuya trama podría distinguirse, sin dificultades, un componente de otro. Esta concepción, sin embargo, encierra varias dificultades, especialmente por los prejuicios ontológicos que implica. Como se advertirá más adelante, la idea de un híbrido o de una naturaleza dual constituida por una suma en cierto modo aleatoria de elementos naturales y artificiales es confundente desde el punto de vista de sus apuestas metafísicas. Paralelamente desestima un puzzle implícito en su condición que podría describirse de la siguiente manera: la naturaleza de los bioartefactos no puede comprenderse completamente por analogía con los entes vivientes pero tampoco con el marco conceptual que explica el estatuto de los artefactos prototípicos.

\footnotetext{
7 En una dirección cercana, Latour (1994, pp. 59-60) comprende este proceso de domesticación en términos de una extensión de las "relaciones sociales" al ámbito de los no humanos. Esta suerte de ecología internalizada consiste, según este autor, en la intensa socialización, reeducación y reconfiguración de plantas y animales, tan intensa que llega a modificar su forma, su función y su makeup genético. La dependencia que estas entidades poseen respecto a cierto tipo de agenciamiento está reconocida por Latour cuando afirma que la domesticación no puede ser comprendida como el "acceso a un campo material objetivo que existe más allá de lo social" (Latour 1994, p.60).
} 
Ni el esquema explicativo autopoiético (por sí solo) ni el esquema de producción intencional (por sí solo) son suficientes para comprender su condición.

Por un lado, a diferencia de aquella serie de entes que no han sido intervenidos intencionalmente, los bioartefactos manifiestan en su historia algún pasaje legítimamente describible como resultado de selección intencional. Sin embargo, se trata de entes con autonomía y autopoiesis cuyo mantenimiento sólo depende parcialmente de las condiciones contextuales provistas por agentes intencionales. Por otro lado, sus condiciones de emergencia no pueden asimilarse al esquema hilpineano intencionalista de autoría basado en el criterio de dependencia contrafáctica (Hilpinen 1992). Ellos no constituyen lo que podríamos llamar "productos" prototípicos. ${ }^{8}$

En resumen, esta ambigüedad inherente al proceso de emergencia y mantenimiento de los bioartefactos dificulta la postulación de un punto de vista que explique de manera unitaria su naturaleza. En lo que sigue se intentará presentar una serie de posibles vías para responder al puzzle implicado en los bioartefactos. En este contexto podrían destacarse dos grandes interpretaciones. La primera de ellas impugnaría la idea misma de bioartefacto. Si se asume una perspectiva naturalista radical, de orientación reproductivista, no es relevante distinguir entre linajes artefactuales "puros", biológicos "puros" y bioartefactuales, pues todos los linajes se hallan relacionados y condicionados en el marco de un proceso coevolutivo. Los fenómenos de copia y éxito o, en términos más generales, las ideas de variación y de retención selectiva, son suficientes para comprender lo que ocurre en cualquier modalidad de deriva evolutiva; no es necesario apelar a ninguna instancia intencional que otorgue dirección al proceso. Otro argumento que concluye en el rechazo de la noción de bioartefactualidad se apoya en una perspectiva intencionalista que opera a partir de artefactos prototípicos y delimita una clara distinción entre objetos técnicos y objetos naturales. Si se admite que sólo una relación de dependencia contrafáctica entre intención y producto da lugar a artefactualidad genuina, no habría lugar para los bioartefactos en un modelo como éste. En segundo término, habría otras lecturas proclives a aceptar la idea de bioartefacto en clave intencionalista. Entre ellas, se podría distinguir un intencionalismo de corte hilpineano y un intencionalismo deflacionado centrado en el proceso. En las siguientes sub-secciones se reconstruirán y evaluarán estas variantes sin que dicha selección signifique rechazar la plausibilidad de otras alternativas.

\footnotetext{
${ }^{8}$ Nuestro lenguaje cotidiano recoge precisamente esa diferencia: el destornillador y la mesa son productos materiales prototípicos pues han pasado por un proceso de conformación intencional. Resulta un tanto más extraño hablar de ciertos vegetales como "productos" aunque las góndolas de los supermercados los presenten bajo esa categoría. Los alimentos, evidentemente, no constituyen "productos" prototípicos. Sin embargo, es innegable que su presencia actual en cuanto mercancía es resultado de extensos procesos de selección intencional con múltiples variantes (selección en el nivel genético o bien selección de fenotipos).
} 


\subsection{La respuesta intencionalista}

Una alternativa para solucionar el puzzle provisto por los bioartefactos sería imponer una condición de dependencia contrafáctica robusta que permitiera identificar productos bioartefactuales. Esto implicaría contemplar al autor y a sus conceptos como fuente de inteligibilidad de la identidad del objeto dando lugar a una teoría "fuerte" de autoría en el sentido de Hílpinen (1992 y 1993). Aquello que se debería focalizar no son las historias reproductivas de los entes sino las intenciones de un agente que -de algún modo- se han cristalizado en el objeto.

La ventaja de este modelo es que, si fuera aplicable exitosamente, nos ofrecería una noción particularmente robusta de bioartefacto asentada en condiciones sólidas de identidad, las corporizadas en una dependencia contrafáctica entre las intenciones del autor y su producto. El principal déficit de esta vía consiste en que, tal como está formulado en Hilpinen, el modelo autor/obra no puede aplicarse exitosamente al ámbito de los bioartefactos. En primer lugar, la condición hilpineana de aceptación no resulta cumplible dado que, en los procesos de selección de larga duración, el autor raramente puede llegar a contemplar y reconocer su obra como tal. Allí el creador no opera sobre el bioartefacto en sí mismo y sus partes sino sobre el fenotipo de una generación con el fin de afectar a la siguiente generación. Este proceso de selección se muestra resistente a la idea tradicional de autoría pues no es posible indicar una única intención productiva que se haya visto plasmada en un objeto particular cuya captación podría conducir a un momento de "reconocimiento". Por el contrario, se requirieron siglos y siglos de intervenciones selectivas anónimas -la mayoría de ellas dotadas de cierta incertidumbre respecto al producto final a conseguir- para que hoy en día contemos con un tipo bioartefactual particular. En segundo término, las limitaciones conectadas con el sesgo demiúrgico del modelo hilpineano se patentizan más en el caso de los bioartefactos. Sería insensato afirmar que una planta de trigo es, en cuanto tal, una mera "corporización" de intenciones, al menos si entendemos dicha corporización como un sencillo movimiento de traslación de rasgos intendidos al plano de la materia. Si se admitiera tal alternativa se estaría realizando una suerte de salto idealizante que vincularía la mera posesión de un concepto con la creación de un nuevo ente. Algo similar puede afirmarse sobre el linaje "perro policía" - caso específico analizado por Mc Laughlin (2003)-. Por un lado, es evidente que no hay ninguna intervención humana material que haga surgir a la familia bioartefactual "perro policía" en cuanto tal, al menos no en el mismo sentido en que se diseña y produce un martillo o un reactor nuclear. Pero, por otro, la misma existencia de un linaje como el mencionado supone la acción previa de agentes que han realizado selecciones intencionales (cada vez más deliberadas y estratégicas) sobre ejemplares de una determinada clase de entes biológicos. 
En resumen, en el contexto de entes biológicos intencionalmente seleccionados como las mascotas o los cereales no puede hablarse legítimamente de autoría, al menos no en este sentido inflacionado propuesto por Hilpinen para explicar las obras tradicionales. Desde este punto de vista no hay estrictamente bio-artefactos, es decir, no hay proceso autoral entendido en los términos tradicionales. Hay sólo entidades naturales completamente independientes de intención. ${ }^{9}$

Ahora bien, si amparados en este enfoque se eligiera rechazar el vocabulario de autoría, no sería necesario invocar justificaciones intencionalistas de ningún tipo para este ámbito peculiar pues se trataría de entes dotados exclusivamente de funciones biológicas. Pero la dificultad emergente sería que una enorme cantidad de procesos de selección intencional que operaron para la existencia de tales entidades en su forma actual quedarían velados, esto es, se perdería la riqueza propia de ese espacio creativo y de coevolución existente desde el Neolítico. Como contrapartida, se estaría en condiciones de defender una concepción fuerte de autoría aplicable, por supuesto, sólo al territorio de las obras tradicionales, ya sea artísticas o técnicas.

¿Hay, entonces, algún modo de enfrentar el puzzle conceptual de los bioartefactos conservando el vocabulario intencionalista? Ciertamente podría concebirse otra sub-variante, también de espíritu hilpineano, que insistiera en que el grado y la calidad de la agencia intencional operante constituyen un criterio rector fiable para definir algún tipo de artefactualidad. Hay una relación directamente proporcional entre el grado de control y la artefactualidad auténtica. En este sentido sería contraintuitivo negar el contraste existente entre el grado de conocimiento científico y el control sofisticado sobre las formas y condiciones de desarrollo de los organismos genéticamente modificados $(O G M)$ y el nivel de control propio de las prácticas empíricamente fundadas de domesticación de los cereales durante el Neolítico.

De allí que una explicación intencionalista alternativa podría restringir el ámbito de los bioartefactos solamente a aquellos entes biológicos que han sido procesados a través de manipulación genética con intervención directa sobre el ADN. Podría pensarse que en los casos de los OGM contemporáneos hay un grado de control suficiente como para satisfacer las exigencias de la relación de dependencia contrafáctica que caracterizan al vínculo autoral. Si aceptamos que el grado de artefactualidad de un producto está directamente relacionado con el grado de control sobre su estructura final (tanto sobre sus propiedades como sobre sus disposiciones

\footnotetext{
9 Precisamente este argumento presenta Thomasson (2009) cuando afirma: "The most obvious sense in which artifacts may be said to be mind-dependent is that artifacts would not exist were it not for the (mental and physical) activities of humans; they are the products of human work. But even this simple point admits of at least two interpretations: there is the causal point, that the intentional activities of humans are causally responsible for the production of tables and chairs, ships and sirens. If this were the only sense of dependence at issue, it might not be of great philosophical interest, since the intentional activities of humans are also causally responsible for the production of a great many natural objects, such as the plants and animals reared in agriculture" $(2009$, p.194).
} 
y potenciales), entonces se podría argumentar que en la producción de OGM sí hay autoría analogable a la producción de un poema o de un sacacorchos. ${ }^{10} \mathrm{Si}$ se admitiera esta última solución, el resto de los entes biológicos espontáneamente mantenidos o seleccionados intencionalmente a través de métodos tradicionales (que operan sobre los fenotipos singulares) quedaría fuera de ese ámbito.

¿Por qué no restringir, entonces, la aplicación del término "bioartefacto" a los ejemplares de OGM? Si bien en un principio parece una salida razonable dado que sobre ellos tenemos un alto grado de control -que no estaba presente en los procesos de domesticación tradicional-, lo cierto es que en esencia se trata de una acción de intervención sobre las cadenas de causas y efectos naturales que cualitativamente también está realizada en la domesticación neolítica que opera con fenotipos. $\mathrm{Ni}$ el rústico criador neolítico ni el refinado ingeniero genético moderno crean vida, sino que solamente intervienen en algunos puntos específicos de una dinámica inmanente. ${ }^{11}$ En resumen, esta sub-variante argumentativa se sostendría solamente al costo de admitir una arbitraria línea de corte entre productos de selección intencional, lo cual debilitaría la defensa de un modelo intencionalista "puro".

Otra estrategia para enfrentar el problema de la naturaleza de la bioartefactualidad sería rechazar la referencia objetivante del concepto "bioartefacto". Se trataría de un intencionalismo deflacionado centrado en la idea de proceso bioartefactual. En esta línea podría argumentarse que ciertas prácticas culturales tales como la agricultura o la cría de ganado -en cuanto reproducción dirigida a fines- conllevan un cierto grado de control y racionalidad estratégica. Dichos procesos, en sí mismos, son bioartefactuales en la medida en que son composicionales (se trata de sistemas que incluyen materia, energía y entes vivientes, algunos de ellos intencionales) pero los resultados de dichos procesos no son "artefactuales", ni "bioartefactuales"; se trata, sencillamente, de entes biológicos con la única característica adicional, ciertamente relevante, de que han sido objeto de selección intencional programada. En esta perspectiva, una planta de trigo o una manzana no serían, en cuanto tales, bioartefactos. En cambio, el proceso dirigido intencionalmente para la producción de dichos entes sí sería adjetivable como "bioartefactual". Lo mismo podría pensarse acerca de otros ejemplos históricos cruciales de procesos bioartefactuales tales como la producción y el uso de levaduras y vacunas. 12

10 Esta es precisamente la perspectiva de Kohler (1994, pp. 53 ss.), quien sostiene que dichos organismos creados en laboratorios con fines de experimentación bien pueden ser catalogados como "living instruments" y resultan analogables, en tal sentido, a un microscopio o un galvanómetro.

11 El tipo de inmanencia que se pretende destacar en este ámbito es similar a aquella que resalta Simondon (2008) cuando habla de las propiedades inmanentes de ciertos materiales cuyas potencias para funcionar sinérgicamente estuvieron siempre pero sólo emergen y se patentizan cuando se las coloca de un modo singular en el interior de un nuevo sistema técnico.

12 En este mismo sentido de proceso bioartefactual pueden situarse otras intervenciones controladas sobre entes naturales (mayormente con fines de alimentación) tales como la producción de vino, de cerveza, de quesos y yogures. Véase, al respecto, Newell-McLoughlin y Re (2006). 
Este giro desde el producto hacia el proceso (que significa también un abandono del sustantivo bioartefacto en favor de su función adjetivante) resulta sugerente en la medida en que propone reflexionar directamente a partir de la escena poiética en lugar que desde el producto ya finalizado, tal como es usual en el debate contemporáneo. Poner el foco en las prácticas significa, en este caso, comprender a la agricultura como un tipo singular de proceso bio-artificial, o intencionalmente dirigido.

La principal ventaja de esta vía explicativa es que coloca a las prácticas en un primer plano, "historiza" la interrogación sobre los bioartefactos. El acervo de una cultura se patentiza en sus prácticas: un cereal domesticado nos habla tanto de su estructura como de la serie de intereses culturalmente sesgados que los agentes pretendieron cristalizar en su práctica selectiva. Por otra parte, dicho enfoque rescata una dimensión ecológica y poiética que suele perderse de vista en los enfoques intencionalistas y reproductivistas: la que considera el proceso poiético que nuclea a agentes y actantes en un entorno determinado tanto por intenciones como por condiciones estructurales no-intencionales. Así este enfoque decide no centrarse en el producto resultante sino en la lógica productiva que lo gobierna.

Ciertamente sería inadecuado rechazar la idea de que el proceso mismo en el que se insertan los bioartefactos comprende una serie de operaciones estratégicas sobre una trama de cadenas causales naturales. Sin embargo, el énfasis colocado en el proceso bioartefactual podría dejar a los productos mismos en la oscuridad, en el sentido en que no parece haber modo de distinguir entre un producto resultante de un proceso bioartefactual auténtico (aquel en el cual han operado estratégicamente agentes intencionales) y un proceso no bioartefactual (uno que se ha generado sin intervención intencional). En otras palabras: si uno restringe exclusivamente su foco al proceso y desatiende al resultado podría desestimar la diferencia existente entre la totalidad de series causales naturales y el (relativamente) pequeño conjunto de cadenas causales sobre el cual el hombre ejerce intervenciones y procesos selectivos a través de distintas prácticas culturales. Este es, según el punto de vista aquí sugerido, el principal déficit de esta sub-variante intencionalista.

En resumen, los argumentos tratados en esta sección muestran que, dentro de los límites del territorio intencionalista, existen tres vías factibles para interpretar el estatuto ontológico de los bioartefactos. Una primera vía toma como punto de partida ciertas condiciones de artefactualidad "prototípica", basándose en ciertos artefactos tradicionales y, al detectar que los entes domesticados no satisfacen dichas condiciones, concluye que no hay bioartefactos sino solamente entes naturales cuya existencia es sólo causalmente dependiente de las actividades intencionales de los agentes humanos. Una segunda vía afirma que una porción de entes domesticados sí conlleva ciertas características que satisfacen las condiciones robustas de artefactualidad propias del enfoque hilpineano. Entre estos entes, los organismos genéticamente modificados involucran no sólo dependencia causal sino conceptual. La ter- 
cera vía, por su parte, desplaza las condiciones de bioartefactualidad desde el producto hacia el proceso al tiempo que focaliza su atención en las prácticas culturales que controlan dichos fenómenos.

\subsection{La respuesta reproductivista}

Una concepción naturalizada -por ejemplo, Elder (2004 y 2007) - argumentaría que tanto los bioartefactos como los artefactos tradicionales pueden ser subsumidos bajo la categoría de copied kinds. No es necesario diferenciar de manera tajante entre la historia reproductiva de los intelectuales franceses del siglo XX, la de las piedras utilizadas en la cultura achelense y la de los cereales. En cuanto trayectorias todas ellas pueden ser exitosamente explicadas por un modelo reproductivista naturalista con la sola ayuda de las nociones de variación, selección y mecanismo de transmisión de las variaciones y -por supuesto- algún ajuste adicional para flexibilizar su aplicación al ámbito del cambio cultural. ${ }^{13}$ Esta perspectiva procuraría aislar las condiciones ambientales que favorecen el mantenimiento de un linaje de entes e identificar los factores que lo obstaculizan. Aquello que se hace evidente es que mientras el intencionalista concentra su análisis en el ente singular en el estado poiético presente y utiliza la ingeniería inversa para captar los conceptos constituyentes desde los cuales fue originado, el naturalista piensa en términos poblacionales y pone su foco en la dinámica histórica y la lógica del cambio.

Comprendida bajo estos términos, resulta claro que la vía reproductivista no reservaría un lugar ontológico especial para los bioartefactos. La noción adecuada para comprender a las vacas Holstein (pero también a las pelotas de tenis y a los representantes del Tea Party) sería la de copied kind. Esta última noción cuenta con la ventaja de que no requiere apelar a intenciones para ser predicada legítimamente sobre un cierto ente, condición que nos exime de utilizar sin éxito un vocabulario de autoría en sentido fuerte y elimina, además, todos los problemas conceptuales que dicho vocabulario conlleva. En este sentido no habría bioartefactos, sino copied kinds con historias reproductivas ontológicamente tan firmes como la que subyace a la producción de vasijas, sillas y represas de castores.

Otra ventaja del enfoque reproductivista es que tiene mejores posibilidades de explicar los intentos iniciales de acoplamiento al ambiente no dirigidos por intencionalidad estratégica, es decir, cubre mejor aquella serie de intervenciones nointencionales que fueron condicionando el perfil de los primeros productos domesticados (entre ellas, las acciones de plant husbandry que precedieron al control de los cereales). ${ }^{14} \mathrm{El}$ proceso selectivo sobre linajes de organismos se puede llevar a

\footnotetext{
13 En las últimas décadas ha habido múltiples intentos de comprender el cambio tecnológico en términos evolucionistas. Entre ellos se destacan Basalla (1988), Ziman (2000) y Mokyr (1996).

14 Contra las lecturas rupturistas que hablan de una revolución agrícola, la domesticación de los cereales resultó -en rigor- un proceso extremadamente extenso y complejo que involucró estadios previos
} 
cabo exitosamente (es decir, puede mantenerse reproductivamente) aun sin que el agente posea una idea clara y precisa del producto final a conseguir ni de sus propiedades particulares. Las trayectorias de las copied kinds anidadas en entes biológicos se auto-mantienen. Los procesos de domesticación que transformaron la historia de la cultura, de hecho, se comprenden mejor pensando en este tipo de reproducciones. Las investigaciones al respecto (Diamond 1997; Murphy 2007; Zeder 2006) muestran que los resultados de estas prácticas selectivas humanas no fueron completamente intendidos (no estaban impresos en la mente de los hacedores antes de la práctica misma) aunque tampoco sus efectos eran completamente desconocidos. En esa ambigüedad reside el núcleo de este tipo de agencia comprendida en sentido débil.

Ahora bien, el enfoque reproductivista revela sus límites en la medida en que se consideran los siguientes factores. En primer término, como toda perspectiva reproductivista, tiene mejores armas para explicar una historia causal ya desplegada que para iluminar la aparición de una nueva serie. ¿Cómo comprender desde una interpretación exclusivamente reproductivista la emergencia de los OGM durante el siglo pasado? Del primer cerdo que es producto de una modificación a nivel genético, ¿podría decirse que es sencillamente un ejemplar "copiado" que se desprende sin más de la historia reproductiva de los cerdos criados en granjas? ¿Implica dicho ejemplar una mera continuidad en la serie de procesos de selección artificial? No resulta evidente que así sea. Pero incluso si aceptáramos esa continuidad evitando apelar a alguna forma de agencia intencional lo cierto es que habría algo significativo (el cambio en el nivel de intervención agencial, el cual ha pasado desde el fenotipo al genotipo) que se perdería en la explicación del surgimiento de ese nuevo linaje.

En segundo lugar, los bioartefactos se caracterizan precisamente por poseer una historia reproductiva que no es completamente explicable sin referencia a aspectos intencionales. ${ }^{15}$ La historia del trigo, por ejemplo, no es absolutamente independien-

de manipulación pre-agricultural de plantas (plant husbandry) sin presencia de cultivo selectivo y sistemático (Murphy 2007). De hecho, los individuos comenzaron a recolectar y procesar cereales salvajes con fines de alimentación al menos diez milenios antes de cultivarlos. Esta lectura favorece la idea de que no hubo una revolución agrícola sino un continuum de decenas de milenios durante los cuales algunos grupos humanos y ciertas plantas coevolucionaron en una serie de asociaciones mutuamente benéficas. La manipulación de plantas sin cultivo formal incluye técnicas tales como transplantaciones, quema controlada y siembra de semillas salvajes recolectadas. Estas prácticas de manipulación no desencadenaron los cambios genéticos que surgieron luego con las variedades domesticadas. Por el contrario, dichas plantas permanecieron como formas salvajes, no se transformaron en entes dependientes de la agencia humana como sí ocurrió con las especies de cultivos completamente domesticados (Murphy 2007, pp. 30 ss).

15 Frente a esta tesis se podría objetar que ciertas clases de entes bioartefactuales prototípicos como la uva sin semilla (ejemplo de bioartefacto analizado por Sperber 2007) pueden surgir también espontáneamente, es decir, no requieren necesariamente agencia intencional para emerger. En el caso de las 
te de la intervención intencional de agentes humanos. En este punto, un supuesto a reconocer es que deben existir linajes de entes biológicos que no han sido sometidos a procesos de domesticación, ya sea por desinterés humano, ya sea por condiciones biológicas objetivas de las propias especies, o bien por una combinación de ambos elementos. ${ }^{16}$ Pero una vez aceptado esto último (que, en rigor, no involucra problemas graves), uno no puede negar que las intervenciones intencionales no se han aplicado a todos los linajes del mundo natural con la misma pasión e insistencia (ni tampoco, podríamos agregar, con el mismo grado de éxito). Según el punto de vista que aquí se defiende, esta presencia -aunque sea mínima- de aspectos intencionales que caracteriza a cierto tipo de prácticas humanas de domesticación constituye un límite infranqueable para los defensores de una teoría exclusivamente reproductivista. En otras palabras: la enorme plasticidad y potencialidad explicativa de dicho enfoque puede convertirse en su déficit más importante en relación con el objetivo puntual de dar cuenta de la especificidad ontológica de los bioartefactos.

Tal vez el enfoque sperberiano sobre los bioartefactos (Sperber 2007) podría ser comprendido como una sub-variante de planteo reproductivista dotado también de un fuerte espíritu naturalista. La idea fundamental de Sperber es que los "artefactos biológicos" (biological artifacts) resultan de asociaciones mutuamente benéficas entre agentes intencionales y otros entes vivientes en el marco de un proceso de coevolución global. En este sentido, Sperber tiende a homologar al cannabis con las mesas y las sillas, es decir, los entes biológicos seleccionados y los entes artificiales prototípicos: "Computers, robots, and their software are no more prototypical artifacts than cannabis and dogs" (2007, p. 136). El problema central de esta concepción es que la referencia a la coevolución recoge una semejanza trivial entre bioartefactos y artefactos tradicionales: la de que ambos se hallan insertos en historias reproductivas atravesadas por múltiples tipos de agencia y factores causales (lo cual inhabilita cualquier interpretación intencionalista clásica sobre el fenómeno). Pero dicha homología edificada sobre la idea de coevolución no ilumina satisfactoriamente las diferencias entre el sapo, la sanguijuela y la vaca Holstein, ni explica tampoco el grado de sofisticación en el nivel de control disponible en cada caso. En otras palabras, el modelo sperberiano es muy útil a la hora de explicar aspectos antiintencionalistas derivados de un enfoque que pone el acento en la coevolución, pero resulta insuficiente cuando se intenta diferenciar entre los grados de control implícitos en distintas prácticas culturales.

uvas sin semilla, este fenómeno corresponde a la estenospermocarpia, un fenómeno natural en el que hay polinización, fertilización y formación del embrión pero este último aborta tempranamente durante su desarrollo. La posibilidad de esta semejanza superficial entre estos dos entes no elimina la especificidad de cierto tipo de acción estratégica desarrollada por grupos humanos, ni tampoco puede borrar el hecho de que dichos entes disponen de historias reproductivas no asimilables entre sí.

16 Aquí es importante destacar que no todas las especies ofrecen condiciones objetivas para la "domesticabilidad"; sólo algunas de ellas las ofrecen y, de aquellas que lo hacen, el hombre elige y opera sólo sobre una determinada sub-serie. Al respecto véanse Jensen (2002) y Diamond (2002). 


\section{Hacia un concepto deflacionado de bioartefacto}

Cada una de las anteriores aproximaciones al fenómeno de los bioartefactos recoge algún aspecto valioso de su modo de ser y de su particular despliegue. No obstante, se señalaron algunas limitaciones que tales enfoques conllevan en la comprensión de dichos entes. Por un lado, contra el reproductivismo radicalizado, se debe admitir que es necesaria cierta referencia mínima a intenciones para explicar la naturaleza de los bioartefactos. Sin alguna clase de intervención intencional capaz de redireccionar la estructura de los linajes de ciertos organismos no habría nada inteligible bajo tal rótulo. Por otro lado, el intencionalismo en su versión hilpineana tiene serias dificultades para dar cuenta del tipo de proceso coevolutivo que involucran los bioartefactos. Es evidente que la idea de un control de tipo demiúrgico sobre la totalidad de rasgos y disposiciones finales del objeto se halla debilitada por la singularidad de los entes bioartefactuales -en verdad no sólo en ellos, pero lo cierto es que en ese ámbito se hacen más evidentes sus déficits-.

Ahora bien, más allá de sus limitaciones hay algunos elementos de la vía de análisis intencionalista que podrían ser preservados con el objeto de elaborar un concepto deflacionado de bioartefacto. En la perspectiva intencionalista la artefactualidad implicaba intencionalidad estratégica, la cual suponía -a su vez- cierta capacidad de control sobre la acción técnica, es decir, cierta capacidad para evaluar resultados a la luz de los objetivos previamente dispuestos. En este sentido acotado es razonable afirmar que hay una relación directamente proporcional entre el grado de control sobre la acción técnica y el grado de artefactualidad del producto.

El reconocimiento de esta relación entre control y artefactualidad no implica necesariamente ser absorbido dentro de un modelo fuerte de autoría. Hay procesos de coevolución de bioartefactos y agentes humanos caracterizados por un alto grado de control (como el caso de los OGM) y otros con muy bajo grado de control-como cierto trato de crianza de animales, que según Ingold (2000), ni siquiera llegaría al estatuto de "domesticación"--. Aun en casos como este último (o en casos de prácticas proto-domesticadoras como la plant husbandry) es innegable la relevancia de algún tipo de agencia, aunque sea superficial, en el que se apoye la detección del éxito y la preservación de la operación que condujo al éxito sin que esto suponga un conocimiento pleno de los aspectos causales involucrados. En otras palabras, si uno pretende defender la relevancia de ciertos momentos de agencia como factores coadyuvantes a la emergencia de bioartefactos se requiere suponer en los agentes la capacidad de evaluar los resultados de la acción técnica, que puedan distinguir entre una acción exitosa y otra que ha fracasado, y que posteriormente sean capaces de reproducir aquella serie de procesos que han evaluado positivamente. 


\subsection{Niveles de intervención intencional}

Si la intervención intencional -de acuerdo con lo argumentado- cumple un papel en la conformación de los bioartefactos en cuanto territorio ontológico, uno de los compromisos teóricos que surge es el de caracterizar tal tipo de intervención de manera suficientemente precisa. A lo largo de este escrito se ha insistido en que es posible ejercer distintos grados de intervención intencional sobre la dinámica inmanente de la naturaleza. En la medida en que el tipo de agencia subyacente a dicha intervención puede servir de guía para señalar aspectos relevantes de la bioartefactualidad, la cuestión que direcciona esta sección es qué grado de intervención es necesario para que pueda hablarse legítimamente de bioartefacto.

En principio habría que descartar todos aquellos subproductos de acciones intencionales, tales como el smog, el agujero en la capa de ozono, la deforestación o la extinción de ciertas especies animales y vegetales. Éstas constituyen consecuencias no pretendidas (aunque no siempre inesperadas) de ciertas acciones técnicas humanas. En verdad, las intervenciones que resultan relevantes para el análisis presentado aquí son las intencionales, esto es, aquellas en las cuales los resultados tienen valor normativo puesto que son evaluados en relación con los objetivos dispuestos.

Una vez hecha esta restricción veamos si los siguientes ejemplos pueden precisar de algún modo los distintos niveles de dichas intervenciones y sus umbrales.

a. Selección de trigo con semillas no auto-esparcibles.

b. Selección de una piedra para ser utilizada como pisapapeles.

c. Selección de un ciervo para ser cazado y luego consumido.

d. Agricultura y cría de animales (a través de selección sobre fenotipos).

e. Organismos genéticamente modificados.

En el caso (a) -que bien puede ser situado históricamente en el contexto previo a la domesticación de los cereales-, el tipo de operación involucrada es selectiva, pero no intencional; sencillamente responde a que los linajes que efectivamente se plantaron fueron aquellos que tenían la disposición objetiva para esa práctica, es decir, aquellas variantes con semillas no auto-esparcibles que quedaban adheridas a la espiga (Preston 2008). Siguiendo a Preston, la explicación de casos como éste no requiere aludir a ninguna instancia de selección intencional; se trataría de un tipo de selección no-intencional. 17

${ }^{17}$ Aquí cabe destacar que, al radicalizar la exigencia del grado de control sobre el producto, el enfoque reproductivista de Preston (2008) termina, curiosamente, afianzando un criterio de bioartefactualidad de clara orientación intencionalista. Preston enfatiza la distinción entre selección intencional y no-intencional y remarca que lo que diferencia a la primera de ellas es la aparición de la genética men- 
CUADRO 1

Niveles de intervención

\begin{tabular}{|c|c|c|}
\hline \multirow{2}{*}{$\begin{array}{l}\text { A. } \\
\text { ENTIDADES } \\
\text { MODIFICADAS } \\
\text { pero no intervenidas } 0 \\
\text { producidas } \\
\text { intencionalmente }\end{array}$} & Ámbito de las entidades inertes & $\begin{array}{l}\text { Ejemplos: } \\
\text { agujero en capa de ozono, } \\
\text { smog. }\end{array}$ \\
\hline & Ámbito de los organismos & $\begin{array}{l}\text { Ejemplos: } \\
\text { deforestación, extinción de } \\
\text { ciertas especies } \\
\text { animales y vegetales. }\end{array}$ \\
\hline \multirow[t]{3}{*}{$\begin{array}{l}\text { B. } \\
\text { ENTIDADES } \\
\text { INTERVENIDAS o } \\
\text { producidas } \\
\text { INTENCIONALMENTE }\end{array}$} & ENTIDADES INERTES & $\begin{array}{l}\text {-Una piedra afilada para ser utilizada } \\
\text { como instrumento cortante. } \\
\text {-Un teléfono móvil. }\end{array}$ \\
\hline & $\begin{array}{l}\text { ORGANISMOS } \\
\text { (dotados de dinámica } \\
\text { autopoiética autónoma) }\end{array}$ & $\begin{array}{l}\text { ORGANISMOS instrumentalizados } \\
\text {-Mediaciones usadas } \\
\text { espontáneamente y sin posterior } \\
\text { reproducción. }\end{array}$ \\
\hline & & $\begin{array}{l}\text { ORGANISMOS DOMESTICADOS } \\
\text { Selección a nivel: } \\
\text {-fenotipo (menor grado de } \\
\text { artefactualidad). } \\
\text {-genotipo (mayor grado de } \\
\text { artefactualidad). } \\
\qquad \text { BIOARTEFACTOS }\end{array}$ \\
\hline
\end{tabular}

En el caso (b) la selección no opera sobre un ente viviente. En verdad, sólo se utilizan las propiedades causales de la piedra. En tal sentido es obvio que no podemos esperar que la trayectoria de esa piedra o de otras "piedra-pisapapeles" se auto-

deliana. Todas las selecciones previas a la genética mendeliana serían no-intencionales. El umbral de control exigido para determinar el carácter bioartefactual de un proceso parece ser aquí demasiado alto. Y aquello que se pierde es el potencial conformador del know-how de los criadores pre-mendelianos plasmado en sus prácticas virtuosas y su notable éxito reproductivo. 
mantenga. El caso (c) se asemeja al anterior en la medida en que la intervención intencional se limita a aprovechar propiedades del ciervo que son insertadas en el marco de propósitos humanos (el ciervo como "producto" alimentario). No se trata de un uso ocasional sino que, por el contrario, es razonable suponer que se trata de una práctica estabilizada dentro del grupo social y condicionada, en cuanto alternativa de alimentación, por las peculiaridades de su propio nicho. Pero hay una diferencia relevante respecto al caso anterior: se trata de una intervención sobre un ser viviente, aunque no haya selección intencional de propiedades mediante procedimientos de cría que puedan inscribir la acción en un proceso de domesticación.

Los casos (d) y (e), por último, representan los ejemplos prototípicos de bioartefacto. En ellos hallamos selección intencional, inserción de los objetos en planes de acción y, lo más importante, podemos narrar sobre ellos una historia reproductiva dirigida por intereses humanos. En este último aspecto se descubren las diferencias relevantes entre una vaca de raza Holstein y otro organismo cuyo linaje no ha sido sometido a selección intencional estratégica.

En resumen, el esclarecimiento de las condiciones de identidad de los bioartefactos requiere aludir a intervenciones intencionales estratégicas realizadas repetidamente sobre linajes de organismos vivos. Considerando lo anterior, un modo de reforzar esta noción de bioartefactualidad es indagar qué tipo de dinámica es exactamente aquella sobre la cual opera la intervención intencional de los agentes humanos.

\subsection{La conexión entre capacidad autopoiética y selección intencional}

En esta instancia resulta lícito dudar acerca de si esta gradualidad creciente de modos de intervención intencional justifica, en verdad, el uso del concepto mismo de "bioartefacto". Dicho de otro modo: ¿es relevante elaborar una noción de bioartefacto tal que refleje de manera apropiada esta diferencia entre el estatuto de dichas prácticas? Una objeción al esfuerzo de articular una noción coherente de bioartefacto podría indicar la conveniencia de iluminar indirectamente tales matices por medio de ajustes en la misma noción de "intervención intencional". Sin embargo, como se mostró en la sección anterior, la intervención intencional por sí sola no funciona adecuadamente como criterio de bioartefactualidad en la medida en que resulta insuficiente si no se conecta de algún modo relevante con el espacio natural intervenido.

El hecho de que los procesos bioartefactuales impliquen una relación entre una serie de organismos y ciertos agentes intencionales no es algo que genere demasiadas dificultades de interpretación. La cuestión espinosa es determinar qué tipo de relación constituyen. En los ejemplos presentados a lo largo de este trabajo se ha visto cómo la emergencia de bioartefactos está relacionada con (1) cierta capacidad autopoiética de los organismos, y (2) ciertos procesos de selección intencional lle- 
vados adelante por agentes. Un concepto valioso de bioartefacto debería ser capaz de articular de modo coherente estos dos aspectos, distinguir ese tipo de relación tal y como aparece en los bioartefactos y mostrar cómo no resulta aplicable en otros casos.

Por un lado, puede haber selecciones intencionales que operan sobre entes sin capacidad autopoiética, por ejemplo, la práctica de seleccionar una serie de piedras de la orilla para armar un dique. El proceso desplegado allí no puede ser denominado bioartefactual. Por otra parte, puede haber trayectorias de ciertos linajes de organismos con capacidad autopoiética que no son interceptadas por ningún proceso de selección intencional, por ejemplo, animales muy alejados de entornos humanos. De modo que tampoco allí podría imputarse la presencia de procesos bioartefactuales.

Esto significa que para que podamos hablar de proceso bioartefactual se requiere que la capacidad autopoiética de cierta clase de organismos se vea "saboteada" por intereses humanos, es decir, direccionada efectivamente por selecciones intencionales realizadas con el objetivo de alcanzar ciertos fines. La conexión entre la intervención y la capacidad autopoiética de los organismos sobre los cuales se opera revela que la agencia genera una diferencia que es necesaria pero no suficiente. Esto es, la intervención intencional es necesaria para desencadenar procesos bioartefactuales, pero no es suficiente dado que requiere operar sobre una dinámica natural inmanente, a saber, la capacidad autopoiética del organismo; sin esta última, la intervención intencional caería al vacío, no se cristalizaría. Sin la capacidad autoreproductiva de los cereales, por ejemplo, los esfuerzos humanos para su domesticación no habrían prosperado.

En otras palabras, la capacidad autopoiética de los organismos que son capaces de devenir bioartefactos y el conjunto de selecciones intencionales realizadas por los agentes no son caminos que corran separados. Operamos sobre ciertas estabilidades inmanentes de los organismos (usufructuando su dinámica autopoiética) mientras que estos últimos aprovechan a su vez la tendencia de los agentes intencionales humanos para alterar ciertos linajes biológicos $-\mathrm{y}$ así dichos organismos logran reproducirse-. Es en el interior de este "círculo virtuoso" de aprovechamientos y relaciones simbióticas donde emergen los procesos bioartefactuales.

\subsection{Condiciones necesarias de bioartefactualidad}

De acuerdo con lo discutido en la sección anterior, la acción intencional es insuficiente para caracterizar y localizar lo bioartefactual. La atribución de bioartefactualidad, por tanto, requiere una especificidad mayor. Una manera de justificar la necesidad de esta búsqueda es pensar la contingencia de la bioartefactualidad de ciertos linajes. La atribución válida de bioartefactualidad a un linaje de organismos puede modificarse a lo largo del tiempo. Algunos linajes de entes pueden haber esta- 
do bajo procesos de selección intencional en algún momento histórico y, sin embargo, posteriormente pueden haber perdido ese estatuto. Otros, por el contrario, pueden haberlo adquirido transitoriamente o de manera estable. El hecho de que actualmente la vaca Holstein pueda ser incluida en el territorio ontológico de los bioartefactos y los sapos se encuentren fuera de él es sólo una condición contingente. Imaginemos que, en un futuro cercano, se descubre que cierto tipo de sapo $M$ (es decir, una cierta variedad de estos organismos) resulta claramente eficaz para curar cierta enfermedad, de manera tal que se favorece artificialmente la reproducción de esta sub-clase en particular. Pasado cierto tiempo, esos sapos pasarían a tener una historia reproductiva dirigida por intereses humanos tal como sucede con la historia reproductiva de los cereales o de ciertas mascotas. Es decir, podríamos presentar un relato de su linaje que incluyera en alguno de sus pasajes alguna referencia a propósitos humanos, propósitos que -a su vez- podrían descubrirse indicialmente en ciertos rasgos de la estructura actual o las disposiciones de dicho organismo. Contra el intencionalismo de raíz hilpineana, la tarea de determinar que algo sea (o no) un bioartefacto tiene que ver con su historia reproductiva, no con la captación inmediata de una esencia o estructura ontológica singular -plasmada en un momento genético de "autoría" - que resultara accesible a través de distintos medios y que fuera captable independientemente de su despliegue reproductivo en el interior de un linaje. 18

De acuerdo con este argumento, el hecho de ser un producto de selección intencional y disponer de una historia reproductiva dirigida intencionalmente son dos aspectos relevantes de lo que significa ser bioartefacto. A esto debería agregarse otra condición necesaria, aunque no suficiente: que se trate de un ente biológico sobre el cual se puedan realizar atribuciones válidas de funciones biológicas y artefactuales. A fin de evitar algunas de las implicaciones problemáticas de estos dos tipos de funciones es razonable recurrir a la terminología propuesta por Lewens (2004). En este sentido, la referencia a los bioartefactos en términos de funciones intendidas y funciones evolutivas habilita a realizar atribuciones válidas de ambos tipos sin que resulten lecturas contradictorias. Si se focaliza el análisis en el ejemplar individual (por ejemplo, esta vaca Holstein) es legítimo realizar atribuciones de funciones intendidas, esto es, pensar en los problemas de "diseño" enfrentados por el "diseñador" y en cómo se hallaron ciertas soluciones a través de selección artificial que condujeron al reforzamiento de un cierto rasgo que posee interés práctico para los agentes involucrados (en el caso analizado, su capacidad para producir más leche). Si se focaliza, en cambio, el plano poblacional, es legítimo atribuir al mismo rasgo de dicha clase de vacas un tipo de función evolutiva. Esto significa que la posesión de ese rasgo en particular favoreció el mantenimiento de ciertos ejempla-

18 Aquí podría arriesgarse la idea de que los bioartefactos -al igual que los artefactos tradicionales según Broncano (2012)- no tienen esencia, sino historia. 
res que disponían de ese rasgo. Estos dos planos de análisis funcional (funciones intendidas y evolutivas) no son contradictorios sino complementarios.

\section{Consideraciones finales}

Este trabajo intentó realizar algunas precisiones sobre el puzzle implícito en la naturaleza de los bioartefactos. El recorrido sugerido por las vías intencionalista y reproductivista destacó la tensión inherente al despliegue de linajes bioartefactuales, tensión que resulta en parte de las singularidades de dichos entes en contraste con los objetos técnicos prototípicos.

Como se ha visto, el intencionalismo de raíz hilpineana no puede explicar satisfactoriamente el hecho de que la disposición final de un bioartefacto no responde a un diseño o a una serie de rasgos intendidos que preexisten en la mente de un "diseñador". En otras palabras, dichos entes no se acoplan bien a un modelo de autoría fuerte. Por su parte, el reproductivismo anti-intencionalista sólo puede responder a los contraejemplos (entre ellos, los casos de emergencia o redireccionamiento de nuevos linajes) si admite alguna instancia de agencia mínima relevante, un punto inicial de acción intencional que inaugura una trayectoria identificable.

En el marco de este puzzle, el presente trabajo caracterizó de manera preliminar un concepto de bioartefacto que intenta pensar más allá de la imagen de un híbrido formado por componentes naturales y artificiales. La principal estrategia fue desplazar el núcleo de indagación del bioartefacto como objeto resultante (individual y aislado) al proceso en sí mismo y a los distintos niveles de intervención intencional por parte de agentes humanos. En este contexto se plantearon ciertas condiciones propias de los bioartefactos, en especial la conexión que debe hallarse entre la capacidad autopoiética de los organismos y las instancias de selección intencional. La relevancia del concepto de bioartefacto radica en que describe ciertas prácticas relacionadas con el diseño técnico, aunque éste se apoye en entidades naturales autopoiéticas que habitualmente no son consideradas como un espacio intervenido intencionalmente.

En resumen, aquí se ha remarcado que es significativo elaborar -en el interior del vocabulario de la filosofía de los artefactos técnicos- una noción deflacionada de bioartefacto que sea capaz de alumbrar una distinción valiosa entre aquellos procesos surgidos de una dinámica natural no intervenida intencionalmente y aquellos resultantes de una intervención intencional sobre dicha dinámica, entes cuya emergencia se da en el interior de una práctica colectiva dirigida por intereses singulares. Se trata, como se ha visto, de una región inestable, constituida en el flujo de nuestras prácticas, sometida a las transformaciones de nuestros potenciales técnicos $\mathrm{y}$, por ello mismo, abierta al ingreso y egreso de ejemplares. 


\section{Referencias bibliográficas}

Basalla, G. (1988): The Evolution of Technology. Cambridge, Cambridge University Press.

BroncAno, F. (2012): La estrategia del simbionte. Cultura material para nuevas humanidades, Salamanca, Ed. Delirio.

Cuevas, A. (2008): "Los bioartefactos: viejas realidades que plantean nuevos problemas en la adscripción funcional", Argumentos de Razón Técnica, n¹1, pp. 71-96.

Diamond, J. (2002): "Evolution, Consequences and Future of Plant and Animal Domestication", Nature, 418, pp. 700-707.

Diamond, J. (1997): Guns, germs and steel. New York, Norton and Company.

Elder, C. (2004): Real natures and familiar objects, Cambridge (Mass.), MIT Press.

ELDER, C. (2007): "On the place of artifacts on ontology", en E.Margolis y S. Laurence (eds.), Creations of the mind. Theories of artifacts and their representation. New York, Oxford University Press.

FEHÉR, M. (1998): “Lo natural y lo artificial”, Teorema, XVII / 3.

Hilpinen, R. (1992): “On artifacts and works of art”, Theoria, (58), 1, pp. 58-82.

Hilpinen, R. (1993), "Authors and Artifacts", Proceedings of the Aristotelian Society, no 93 , pp. 155-178.

InGOLD, T. (2000): The perception of the enviroment. Essays on livelihood, dwelling and skill, New York, Routledge.

Jensen, P. (2002): "Behaviour Genetics, Evolution and Domestication", en P. Jensen (ed.), The Ethology of Domestic Animals, Wallingford, CABI Pub.

KoHLER, R. (1994): Lords of the Fly: Drosophila genetics and the experimental life. Chicago, The university of Chicago Press.

LAtour, B. (1994): "On technical mediation. Philosophy, sociology, genealogy", Common knowledge, (3), 2, pp. 29-64.

LEE, K. (2009): "Biology and technology", en J.Olsen y otros (eds.), A Companion to the Philosophy of Technology, Oxford, Willey-Blackwell.

LEWENS, T. (2004): Organisms and artifacts. Design in nature and elsewhere, Cambridge, MIT Press.

LONGY, F. (2009): "How biological, cultural and intented functions combine", en U.Krohs y P.Kroes (eds.), Functions in biological and artificial worlds, Londres, MIT Press.

Mc Laughlin, P. (2003): What functions explain. Functional explanation and selfreproducing systems, New York, Cambridge University Press.

MOKYR, J. (1996): "Evolution and technological change: a new metaphor for economic history", en R.Fox (Ed.), Technological Change, Londres, Harwood Publishers. 
Murphy, D. (2007): People, plants, and genes. The Story of crops and humanity, New York, Oxford University Press.

Newell-McGloughlin, M. y RE, E. (2006): The evolution of biotechnology, Londres, Springer.

Preston, B. (2008): “The Shrinkage factor: comment on Lynne Rudder Baker's The Shrinking difference between artifacts and natural objects", $A P A$ Newsletters, Vol 8, Number 1.

Simondon, G. (2008): El modo de existencia de los objetos técnicos, Buenos Aires, Prometeo.

Sperber, D. (2007): "Seedless grapes: nature and culture", en E.Margolis y S.Laurence (eds.), Creations of the mind. Theories of artifacts and their representation, New York, Oxford University Press.

Thomasson, A. (2009): "Artefacts in metaphysics", en A.Meijers (ed.), Philosophy of technology and engineering sciences, Amsterdam, Elsevier B.V.

Vera, C., Blu, A. y Torres, M. (2005): "Sanguijuelas, parásitos presentes ayer y hoy", Revista Chilena de Infectología, 22 (1): pp. 32-37.

ZEDER, M. y otros, eds. (2006): Documenting domestication. Los Angeles, University of Carolina Press.

Ziman, J. (ed.) (2000): Technological Innovation as an Evolutionary Process, Cambridge, Cambridge University Press.

Diego Parente

Universidad Nacional de Mar del Plata - CONICET, Argentina diegocparente@yahoo.com 